\title{
Clinical significance of microRNA-155 expression in hepatocellular carcinoma
}

\author{
CHENGNONG GUAN ${ }^{1,2}$, FENG YANG ${ }^{2}$, XICHUN HE $^{2}$, TUHUA LI $^{2}$, \\ QINGMEI YANG ${ }^{2}$, HUIPING $\mathrm{HE}^{2}$ and MENG XU ${ }^{1}$ \\ ${ }^{1}$ Department of Oncology, The First Affiliated Hospital of Jinan University, Guangzhou, Guangdong 510630; \\ ${ }^{2}$ Center of Oncology, The Affiliated Hospital of Guangdong Medical College, Zhanjiang, Guangdong 524000, P.R. China
}

Received December 31, 2014; Accepted July 30, 2015

DOI: $10.3892 / \mathrm{ol} .2015 .4048$

\begin{abstract}
The present study aimed to evaluate the expression of microRNA-155 (miR-155) in hepatocellular carcinoma (HCC) and adjacent normal tissues, and assess its correlation with clinicopathological characteristics of this tumor type. miR-155 expression was detected in 40 HCC tissue samples and 40 samples of adjacent tumor-free tissue using fluorescent reverse transcription-quantitative polymerase chain reaction (RT-qPCR). The association between miR-155 expression, clinicopathological features and 1-year relapse-free survival (RFS) in HCC and adjacent normal tissue samples was analyzed. RT-qPCR results revealed that, in 25 cases $(62.5 \%)$, miR-155 expression levels were significantly increased in HCC tissues compared with the expression levels observed in pericarcinomatous tissues $(\mathrm{P}<0.05)$. miR-155 expression was observed to be significantly correlated with vessel invasion, Edmonson classification and clinical stage $(\mathrm{P}<0.05)$. However, miR-155 expression was not significantly correlated with gender, age, tumor size, tumor number, hepatitis B virus DNA copy number, cirrhosis or concentration of $\alpha$-fetoprotein $(\mathrm{P}>0.05)$. A positive correlation was observed between late TNM classification of malignant tumor stage and 1-year RFS $(\mathrm{P}<0.05)$. Patients exhibiting high miR-155 expression levels were observed to exhibit a lower 1-year RFS than that of patients with reduced expression of miR-155 (48 vs. 73.3\%), however this difference was not statistically significant $(\mathrm{P}=0.105)$. Additionally, correlations were observed between miR-155 expression and reduced differentiation, increased invasiveness and late stages of HCC. The current results demonstrated that miR-155 may be involved in the tumorigenesis of HCC and may be associated with clinical
\end{abstract}

Correspondence to: Mr. Meng Xu, Department of Oncology, The First Affiliated Hospital of Jinan University, 613 Huangpu Dadao Xi, Guangzhou, Guangdong 510630, P.R. China E-mail: mengxucn@163.com

Key words: microRNA-155, relapse-free survival, reverse transcription-quantitative polymerase chain reaction, hepatocellular carcinoma characteristics of HCC patients. Additional studies are required to clarify the mechanism of miR-155.

\section{Introduction}

Hepatocellular carcinoma (HCC) is a major malignant tumor, which currently represents a significant threat to human health. Worldwide, HCC is the fifth most common malignant tumor observed in males, and the seventh most common amongst females. Furthermore, HCC is ranked second and sixth in terms of mortality for males and females, respectively (1). Approximately $50 \%$ of all novel HCC cases were diagnosed in China in 2008 (2). In China, liver cancer is the second largest cause of mortality in terms of malignant tumors. In 2008 , only $30 \%$ of patients in Western countries and $>10 \%$ of patients in Asia were eligible for curative therapies, including surgery orthotopic liver transplant or local ablation. Palliative treatments include transcatheter arterial chemoembolization and sorafenib (3). However, the majority of HCC patients are diagnosed at a late stage, due to the lack of highly specific and sensitive methods for the diagnosis of HCC in its early stages (4). Thus, $\sim 80 \%$ of patients with HCC in China are diagnosed at a late stage, and the situation with regard to the diagnosis and treatment of liver cancer remains a significant issue (5). Previous studies have investigated the mechanisms underlying the occurrence and development of HCC; however, as yet, these mechanisms remain to be fully elucidated $(6,7)$. Therefore, investigation of the pathogenesis of HCC, as well as exploration of novel diagnostic markers and therapeutic targets, have important significance for improving the diagnosis and treatment of HCC.

The first microRNA (miRNA) was identified in Caenorhabditis. elegans in 1993. miRNAs are small, single stranded non-coding RNAs of $\sim 18-25 \mathrm{nt}$ in length (8). miRNAs combine with the 3'-untranslated region of target messenger RNA (mRNA), resulting in translational repression or mRNA degradation. miRNAs are involved in various physiological and pathological processes, including cell proliferation, differentiation, growth, development and tumorigenesis (9). microRNA-155 (miR-155) is located on the third exon of the B-cell integration cluster (BIC) gene of human chromosome 21 (10). BIC does not contain an open reading frame and its overexpression may promote the abnormal 
proliferation of cells. Experimental evidence has indicated that miR-155 is overexpressed in a number of types of neoplastic disease (11-13). miR-155 has a significant role in carcinogenesis, primarily as an oncogene (14).

The present study identified that miRNA-155 has a number of associations with the clinicopathological parameters of tumors. Additionally, miR-155 has an effect on the assessment of disease severity and estimate of prognosis of tumors. A study revealed that increased expression of miR-155 was associated with TNM classification of malignant tumor stage (TNM), lymph node metastasis and proliferating cell nuclear antigen positivity in breast cancer (15). Papaconstantinou et al (16) identified that miR-155 expression was associated with clinical stage and poor prognosis in pancreatic cancer. Additionally, Shibuya et al (17) identified that miR-155 expression was associated with lymph node metastasis in colorectal cancer. A further study revealed that there were significant associations between miR-155 expression and the development of digestive tract cancer (18). However, the association between the clinicopathological features of HCC and miR-155 expression remains to be elucidated. In addition, with the increase in the recurrence rate of HCC, an association between miR-155 expression levels and early recurrence following surgery remains to be elucidated. The current study will present an in-depth investigation of these outstanding questions, by analyzing the expression of miR-155 in HCC tissues and its clinical significance.

\section{Materials and methods}

Specimens. The present study was conducted at the Department of Oncology, The First Affiliated Hospital of Jinan University (Guangzhou, China) and the Department of Oncology, The Affiliated Hospital of Guangdong Medical College (Zhanjiang, China). A total of 40 samples of cancerous and 40 samples of pericarcinomatous $(3-5 \mathrm{~cm}$ from the tumor edge, confirmed by histopathology) tissue were removed from patients exhibiting HCC during surgery between January and December 2012. The patients had not received any other anticancer treatments prior to surgery. The present study was conducted in accordance with the Declaration of Helsinki (19) with approval from the Ethics Committee of Jinan University (Guangzhou, China). Written informed consent was obtained from all participants. The follow-up deadline was 31 December 2012; the shortest follow-up period was 3 months and the longest was 12 months.

Reverse transcription-quantitative polymerase chain reaction (RT-qPCR). To quantitate mRNA expression, total RNA was extracted from clinical samples and NPC cell lines using a cDNA Synthesis Kit (Takara Bio, Inc., Otsu, Japan). The isolated total RNA was reverse transcribed using the One Step PrimeScript miRNA cDNA Synthesis Kit (Takara Bio, Inc.) for miR-155, according to manufacturer's instructions. The sequence-specific forward primers for mature miR-155 and U6 internal control were 5'-ACACTCCAGCTGGGTTAATGCTAATCGTG-3' and 5'-CTCGCTTCGGCAGCACA-3', respectively (Takara Bio, Inc.). qPCR was performed using SYBR Premix ExTaq ${ }^{\mathrm{TM}}$ II (Takara Bio, Inc.) in a LightCycler 480 system (Roche Diagnostics, Indianapolis, IN, USA). The parameters of the PCR reaction were as follows: $94^{\circ} \mathrm{C}$ for $2 \mathrm{~min}, 1$ cycle; $94^{\circ} \mathrm{C}$ for $20 \mathrm{sec}, 60^{\circ} \mathrm{C}$ for $34 \mathrm{sec}$ for 40 cycles. All RT-qPCR reac-

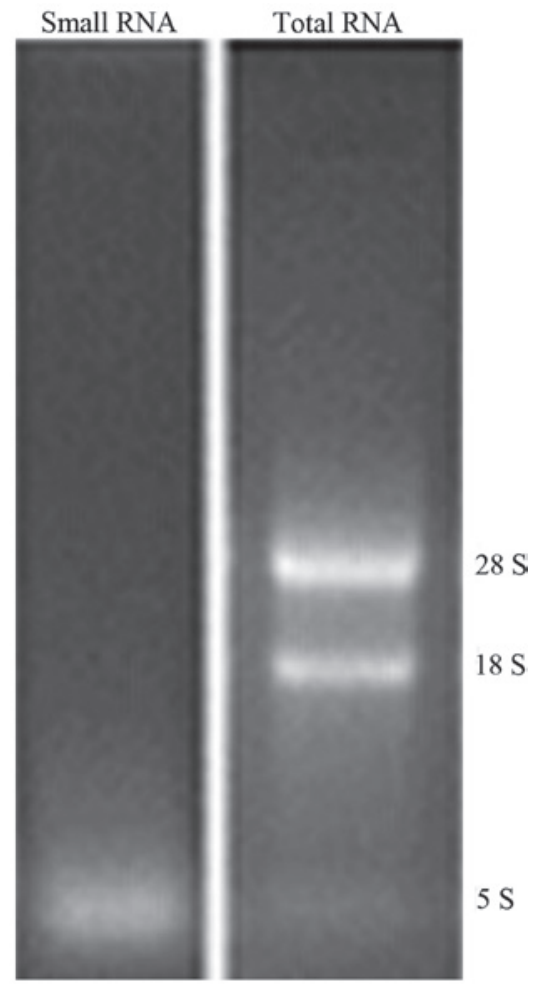

Figure 1. Agarose gel electrophoresis of RNA. Small RNA exhibited a bright $5 \mathrm{~S}$ band, indicating successful miRNA extraction.

tions were performed in triplicate. Relative expression was calculated using comparative cycle threshold $(\mathrm{Ct})$ values. The U6 small nuclear ribonucleoprotein was used as the inner reference gene for miR-155. miR-155 relative expression was calculated using $2^{-\Delta \mathrm{Ct}}, \Delta \mathrm{Ct}=\mathrm{Ct}(\mathrm{miR}-155)-\mathrm{Ct}(\mathrm{U} 6)$. Relative quantification of miR-155 expression in cancer versus pericarcinomatous tissues was calculated using the $2^{-\Delta \Delta \mathrm{Ct}}$ method, $\Delta \Delta \mathrm{Ct}=\Delta \mathrm{Ct}($ experimental group) $-\Delta \mathrm{Ct}$ (control group).

Statistical analysis. Data were analyzed using SPSS version 17.0 (SPSS, Inc., Chicago, IL, USA). Results of measurement data that were not normally distributed are expressed as the median and interquartile range (25-75\%). Comparisons of the expression of miR-155 in cancerous and pericarcinomatous tissues were performed using the Wilcoxon signed rank test. miR-155 expression was compared across a diverse group of clinicopathological features using the Mann-Whitney test. Survival curves were constructed using the Kaplan-Meier method and evaluated using the Log-Rank test. $\mathrm{P}<0.05$ was considered to indicate a statistically significant difference.

\section{Results}

Confirmation of successful miRNA extraction. Small fragments of RNA ( $<200$ bp) were acquired using the miRcute miRNA Isolation kit. Electrophoresis revealed a bright $5 \mathrm{~S}$ band, indicating successful miRNA extraction (Fig. 1).

PCR results indicate miRNA expression. miR-155 expression was detected using SYBR Green fluorescence RT-qPCR. The amplification curves of the target and reference genes 
A

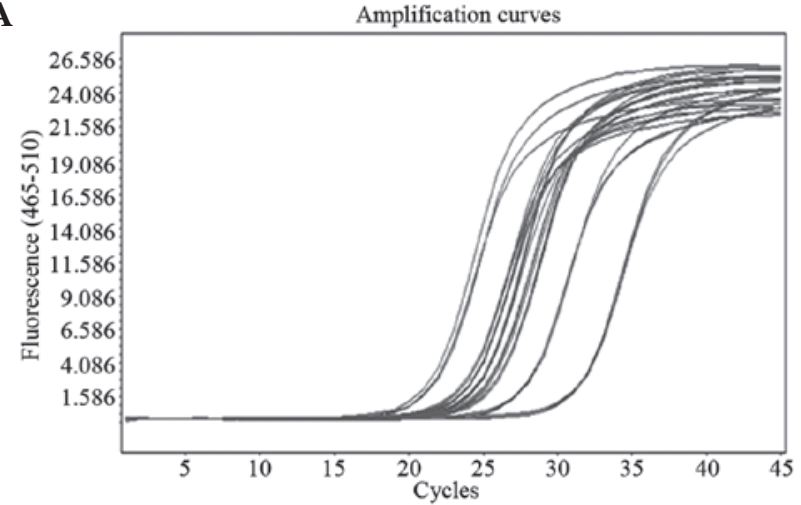

C

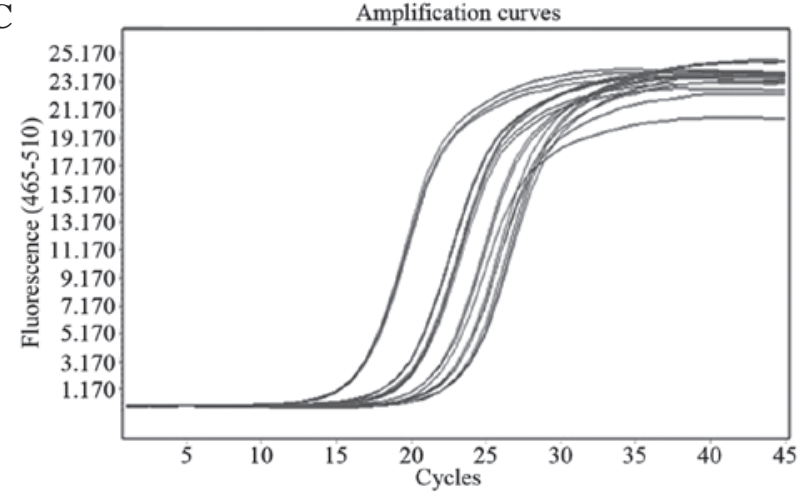

B

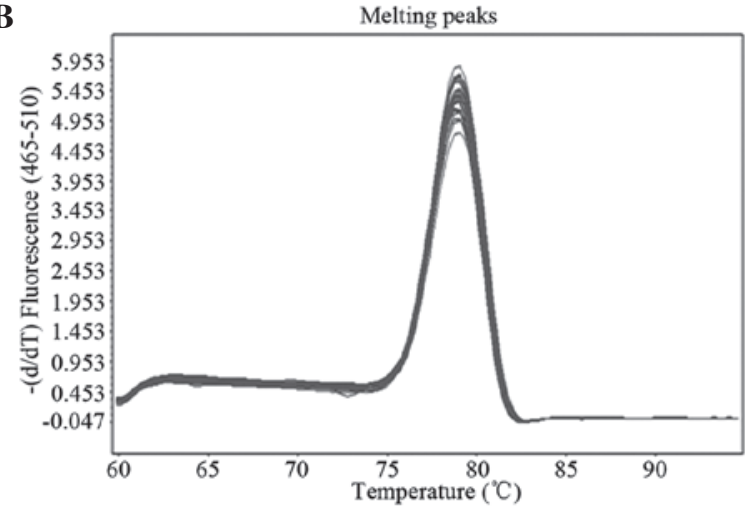

D

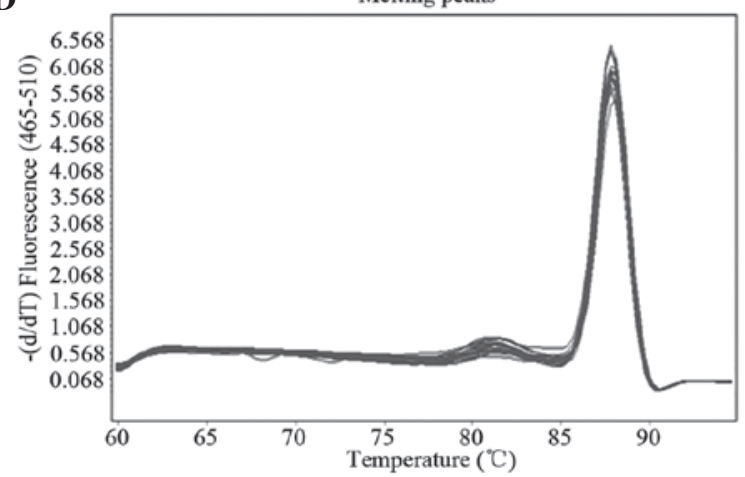

Figure 2. Amplification and dissolution curves. (A) Amplification curve of miR-155; (B) dissolution curve of miR-155; (C) amplification curve of U6 and (D) dissolution curve of U6. miR-155; microRNA-155; d/dt, derivative with respect to t.

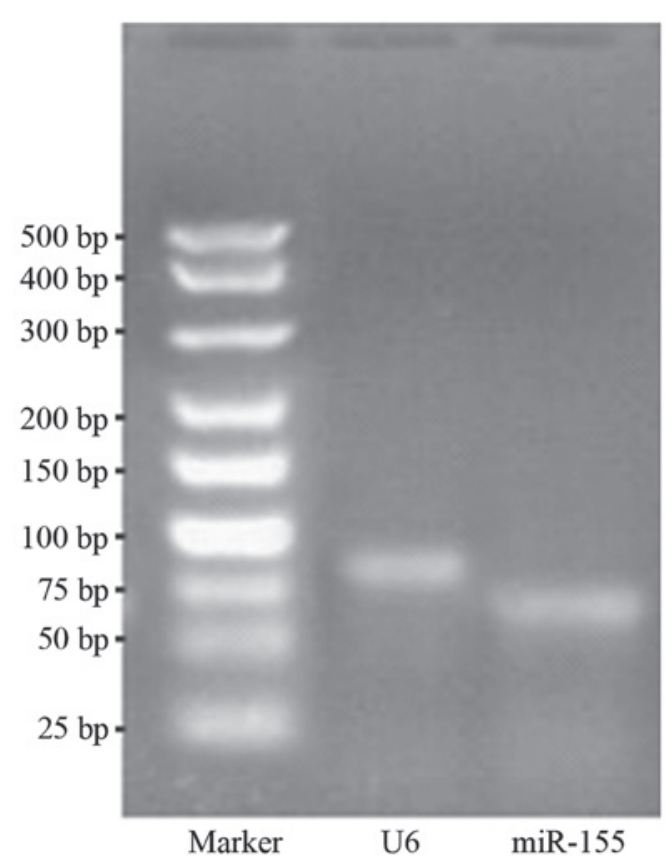

Figure 3. Polymerase chain reaction products evaluated using agarose gel electrophoresis. Two clear cataphoresis bands were visualized at 100 and $75 \mathrm{bp}$, consistent with the theoretical values for the reference gene U6 (94 bp) and target gene miR-155 (70 bp). U6, U6 small nuclear ribonucleoprotein.

were smooth, indicating that the amplification system was suitable for the reaction and the experiment conditions were correct. Thus, suggesting good experiment repeatability. The dissolution curve demonstrated narrow and sharp peaks and no impurity peaks, and the various melting temperatures

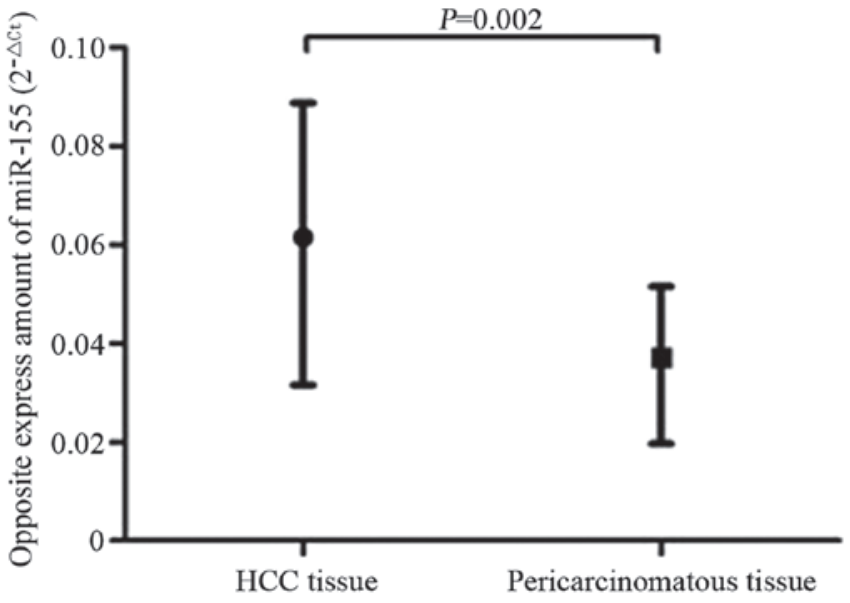

Figure 4. Increased miR-155 expression in HCC tissue. Opposite express quantities of miR-155 in HCC and pericarcinomatous tissue. HCC, hepatocellular carcinoma; miR-155, microRNA-155. Values are expressed as the mean and interquartile range.

identified correspond with distinct gene products. The dissolution temperature for each gene product was comparable in the various samples, confirming the high specificity of the amplified product (Fig. 2).

PCR product analysis with agarose gel electrophoresis. There were two clear cataphoresis bands visualized at 100 and $75 \mathrm{bp}$, which were consistent with the theoretical values for the reference gene U6 small nuclear ribonucleoprotein (94 bp) and target gene miR-155 (70 bp). Additionally, no other impurity 
Table I. Analysis of miR-155 expression levels in HCC and pericarcinomatous tissue.

\begin{tabular}{|c|c|c|c|}
\hline Tissue group & Cases, $\mathrm{n}$ & Relative expression levels of miR-155 & P-value \\
\hline $\mathrm{HCC}$ & 40 & $0.061(0.032-0.089)$ & 0.002 \\
\hline Pericarcinomatous & 40 & $0.037(0.019-0.052)$ & \\
\hline
\end{tabular}

${ }^{\text {aV}}$ Values are expressed as the median (25th-75th percentile). HCC, human hepatocellular carcinoma; miR-155, microRNA-155.

A

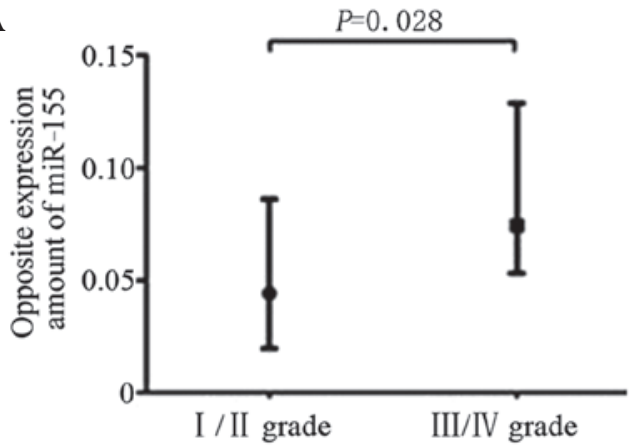

B

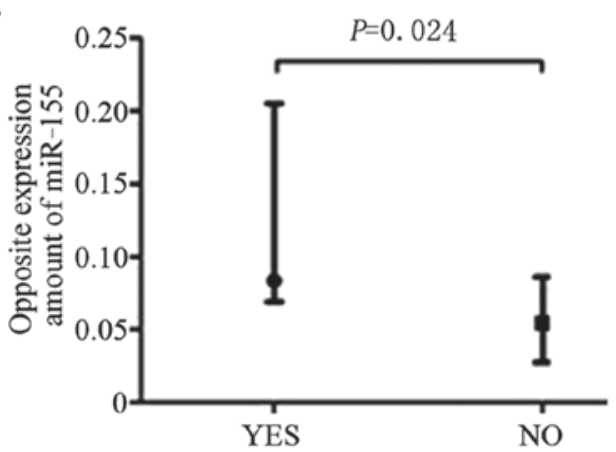

C

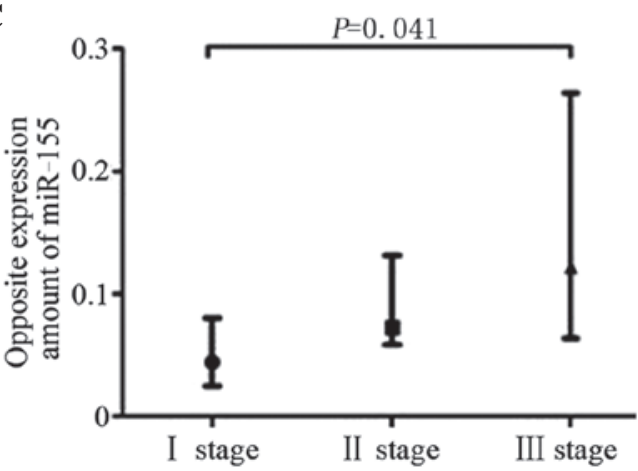

Figure 5. Association between miR-155 expression and (A) Edmonson grade, (B) vascular invasion and (C) American Joint Committee on Cancer TNM classification of malignant tumor stage. miR-155, microRNA-155. Values are expressed as the mean and interquartile range.

bands were observed, indicating that experimental conditions and primer design were correct (Fig. 3).

miR-155 is upregulated in HCC clinical specimens. In HCC tissues, miR-155 expression was upregulated in $62.5 \%(25 / 40)$ of samples, compared with that of pericarcinomatous tissue samples. The mean level of upregulation was a 2.570 -fold increase [95\% confidence interval (CI); 1.928-3.212; Table I].

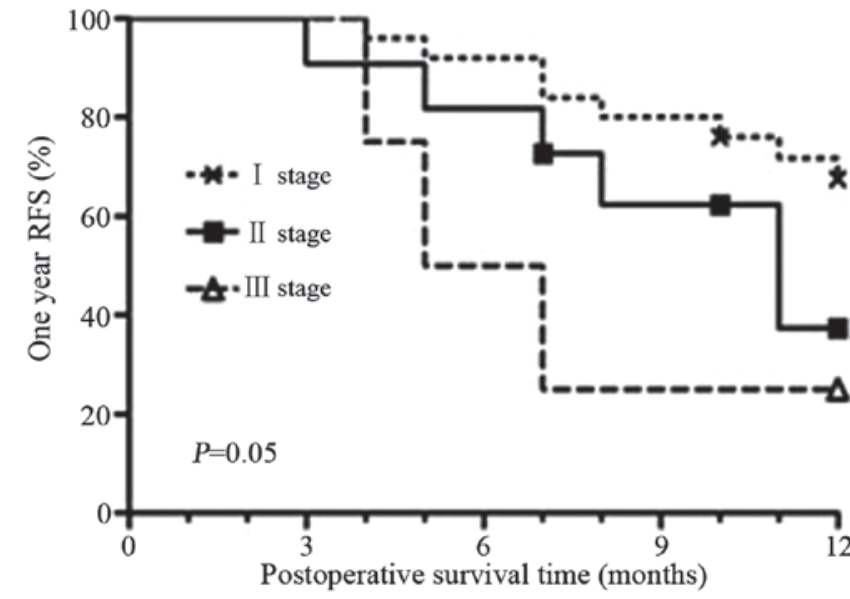

Figure 6. Association between the TNM classification of malignant tumor stage of patients with hepatocellular carcinoma and 1-year RFS. RFS, relapse-free survival.

Thus, miR-155 was significantly upregulated in HCC tissues compared with adjacent normal tissues ( $\mathrm{P}=0.002$; Fig. 4).

Association between miR-155 expression levels and clinicopathological parameters of HCC. An association was observed between miR-155 expression levels and Edmonson grade of HCC tissue, vascular invasion and clinical stage $(\mathrm{P}<0.05)$. However no association was observed between miR-155 expression and the gender, age, tumor size, tumor number or $\alpha$-fetoprotein levels of patients (P>0.05; Table II; Fig. 5).

Association between miR-155 expression levels and 1-year relapse-free survival (RFS). Following surgery, the 40 patients who had exhibited HCC were followed-up for 1 year. Three of these patients were lost to follow-up during the follow-up period, resulting in a follow-up rate of 92.5\%. During follow-up, there were 17 cases of tumor recurrence, therefore total 1-year RFS was $57.5 \%$. The 40 patients evaluated in the present study were divided into high and low miR-155 expression groups. miR-155 expression levels in HCC tissue were classified as high when observed expression levels were $>1$-fold greater than the expression levels observed in pericarcinomatous tissue. By contrast, when expression levels in HCC tissue were observed to be $<1$-fold that observed in percarcinomatous tissue, a classification of low expression was applied. The Kaplan-Meier method was applied for prognostic analysis of single factors using the log-rank test. The result of this analysis revealed that there was a positive correlation between late TNM stage and reduced 1-year RFS $(\mathrm{P}=0.05)$. The percentage of patients with 
Table II. Association between miR-155 expression and clinicopathological parameters of human hepatocellular carcinoma.

\begin{tabular}{|c|c|c|c|}
\hline Clinicopathological parameters & Cases, $\mathrm{n}$ & Relative expression levels of miR-155 & P-value \\
\hline Gender & & & 0.567 \\
\hline Male & 35 & $0.062(0.029-0.089)$ & \\
\hline Female & 5 & $0.058(0.019-0.097)$ & \\
\hline Age, years & & & 1.000 \\
\hline$>50$ & 18 & $0.055(0.035-0.113)$ & \\
\hline$\leq 50$ & 22 & $0.062(0.027-0.087)$ & \\
\hline Edmonson grade & & & $0.028^{b}$ \\
\hline $\mathrm{I} / \mathrm{II}$ & 23 & $0.044(0.019-0.086)$ & \\
\hline III/IV & 17 & $0.074(0.053-0.129)$ & \\
\hline Tumor size, $\mathrm{cm}$ & & & 0.887 \\
\hline$>5$ & 14 & $0.055(0.029-0.095)$ & \\
\hline$\leq 5$ & 26 & $0.062(0.033-0.087)$ & \\
\hline No. of tumors & & & 0.617 \\
\hline 1 & 30 & $0.060(0.039-0.087)$ & \\
\hline$\geq 2$ & 10 & $0.062(0.049-0.133)$ & \\
\hline Vascular invasion & & & $0.024^{b}$ \\
\hline Yes & 6 & $0.083(0.069-0.205)$ & \\
\hline No & 34 & $0.054(0.027-0.086)$ & \\
\hline Cirrhosis & & & 0.325 \\
\hline Yes & 23 & $0.062(0.021-0.086)$ & \\
\hline No & 17 & $0.061(0.042-0.132)$ & \\
\hline Malignant tumor stage $^{c}$ & & & $0.041^{\mathrm{b}}$ \\
\hline I & 25 & $0.044(0.025-0.080)$ & \\
\hline II & 11 & $0.072(0.058-0.131)$ & \\
\hline III & 4 & $0.122(0.063-0.264)$ & \\
\hline Hepatitis-B virus DNA, copies/ml & & & 0.051 \\
\hline$>500$ & 17 & $0.074(0.059-0.099)$ & \\
\hline$\leq 500$ & 23 & $0.044(0.021-0.074)$ & \\
\hline$\alpha$-fetoprotein, $\mathrm{ng} / \mathrm{ml}$ & & & 0.086 \\
\hline$\geq 400$ & 15 & $0.044(0.029-0.069)$ & \\
\hline$<400$ & 25 & $0.074(0.036-0.099)$ & \\
\hline
\end{tabular}

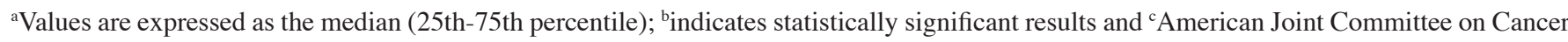
TNM classification. miR-155, microRNA-155.

high miR-155 expression achieving 1-year RFS was reduced compared with that of patients with low miR-155 expression (48.1 vs. $73.3 \%$ ), however this difference was not statistically significant ( $\mathrm{P}=0.118$; Table III; Fig. 6).

\section{Discussion}

The occurrence of primary liver cell cancer is a chronic process, which involves a number of steps, including the inactivation of anti-oncogenic processes, the activation of oncogenes and the abnormal regulation of various cell signaling pathways (20). At present, a key area of liver cancer research is elucidation of the association between miR-155 expression and HCC. The present study revealed that miR-155 expression levels were enhanced in HCC tissues, consistent with previous findings by $\mathrm{Hu}$ et al (21).
The present study also revealed an association between miR-155 expression levels and Edmonson grade (grade III/IV), vascular invasion and clinical stage $(\mathrm{P}<0.05)$, which was consistent with the findings of Song et al (22) in breast cancer. However, the results of the present study were inconsistent with the findings of Han et al (23), where an association between miR-155 expression and HCC tissue differentiation was observed, as the present study did not compare the differential expression in high and low HCC differentiation groups. In the present study, Edmonson grades I/II were classified as one group and grades III/IV were classified as another group. High expression of miR-155 and high Edmonson grade demonstrated a significant association $(\mathrm{P}<0.05)$. These results suggested that high expression levels of miR-155 may be associated with a high degree of malignancy and invasion in HCC. According to 
Table III. Single factor analysis of influencing factors of 1 year RFS.

\begin{tabular}{|c|c|c|c|c|}
\hline \multirow[b]{2}{*}{ Clinicopathological parameters } & \multirow[b]{2}{*}{ Cases, n } & \multirow[b]{2}{*}{ 1-year RFS, \% } & \multicolumn{2}{|c|}{ Log-rank test } \\
\hline & & & $\chi^{2}$ & P-value \\
\hline Gender & & & 1.588 & 0.213 \\
\hline Male & 35 & 60.0 & & \\
\hline Female & 5 & 40.0 & & \\
\hline Age, years & & & 0.819 & 0.374 \\
\hline$>50$ & 18 & 67.0 & & \\
\hline$\leq 50$ & 22 & 50.0 & & \\
\hline Edmonson grade & & & 1.808 & 0.181 \\
\hline $\mathrm{I} / \mathrm{II}$ & 23 & 65.0 & & \\
\hline III/IV & 17 & 47.1 & & \\
\hline Tumor volume, $\mathrm{cm}$ & & & 0.965 & 0.324 \\
\hline$>5$ & 14 & 50.0 & & \\
\hline$\leq 5$ & 26 & 61.5 & & \\
\hline No. of tumors & & & 1.988 & 0.168 \\
\hline 1 & 30 & 63.0 & & \\
\hline$\geq 2$ & 10 & 40.0 & & \\
\hline Vascular invasion & & & 2.709 & 0.100 \\
\hline Yes & 6 & 33.0 & & \\
\hline No & 34 & 61.8 & & \\
\hline Hepatocirrhosis & & & 3.620 & 0.067 \\
\hline Yes & 24 & 46.0 & & \\
\hline No & 16 & 75.0 & & \\
\hline Malignant tumor stage ${ }^{a}$ & & & 6.004 & $0.050^{\mathrm{b}}$ \\
\hline I & 25 & 68.0 & & \\
\hline II & 11 & 46.0 & & \\
\hline III & 4 & 25.0 & & \\
\hline Hepatitis-B surface antigen, copies/ml & & & 2.196 & 0.141 \\
\hline$>500$ & 17 & 47.0 & & \\
\hline$\leq 500$ & 23 & 65.2 & & \\
\hline$\alpha$-fetoprotein, $\mathrm{ng} / \mathrm{ml}$ & & & 1.316 & 0.257 \\
\hline$\geq 400$ & 15 & 47.0 & & \\
\hline$<400$ & 25 & 64.0 & & \\
\hline Postoperative chemotherapy & & & 0.298 & 0.592 \\
\hline Yes & 16 & 62.5 & & \\
\hline No & 24 & 54.2 & & \\
\hline microRNA-155 & & & 2.632 & 0.118 \\
\hline Overexpression & 25 & 48.0 & & \\
\hline Low expression & 15 & 73.3 & & \\
\hline
\end{tabular}

${ }^{a}$ American joint committee on cancer TNM classficiation; bindicates statistically significant results. RFS, relapse-free survival.

these data, miR-155 appears to be involved in tumor progression through the inhibition of multiple tumor suppressor genes, such as sex-determining region Y-gene related high-mobility-group box gene $(24,25)$ and suppressor in cytokine signaling 1 (26), thus promoting proliferation and invasion in HCC. Surgical resection is the typical initial treatment for primary liver cancer (27). This previous demonstrated that the rate of HCC surgery recurrence rate was significantly increased within 1 year of surgery and was accompanied by poor prognosis, compared with the recurrence rate in subsequent years. Therefore, recurrence of $\mathrm{HCC}$ within 1 year following radical surgical resection is defined as early recurrence $(28,29)$. A previous study (29) demonstrated that the total relapse rate in the first year directly following surgery was $43.3 \%$, and $\mathrm{HCC}$ recurrence in subsequent years was $42.5 \%$, which was consistent with the findings of Sun et al (29). Cai et al (30) retrospectively analyzed HCC 
recurrence in 110 patients who had undergone radical surgical resection, and found that the formation of portal vein thrombus was a risk factor for early recurrence. Single factor analysis indicated that the postoperative 1-year RFS of HCC patients exhibiting vascular invasion was reduced compared with that of patients with $\mathrm{HCC}$ and no vascular invasion (33.6 vs. 61.8\%), however a statistically significant difference was not indicated $(\mathrm{P}=0.10)$ and these findings were inconsistent with the results of a previous study (30). A problem with the present study was that the sample size of patients with $\mathrm{HCC}$ and vascular invasion was markedly smaller than the sample with HCC and no vascular invasion. Furthermore, the present study demonstrated that the 1 -year RFS of late TNM stage patients was reduced $(\mathrm{P}=0.05)$.

There is a close association between miR-155 expression levels and HCC prognosis $(31,32)$. Huang et al $(33)$ demonstrated that there was an association between miR-155 expression levels and 5-year RFS of patients with HCC following radical surgical resection. The 5-year RFS of patients expressing high levels of miR-155 was reduced compared with patients exhibiting low levels of miR-155 (hazard ratio=2.002; 95\% CI, 1.324-3.027). It remains to be fully elucidated whether miR-155 is associated with early recurrence of HCC. In the present study, it was observed that 1-year RFS of patients exhibiting high expression of miR-155 was reduced compared with patients exhibiting low expression levels of miR-155 (48.1 vs. 73.3\%), however this difference was not statistically significant $(\mathrm{P}=0.105)$. Therefore, miR-155 expression levels may not be the sole predictor for early recurrence of HCC. Therefore, although miR-155 was overexpressed in HCC patients'tissues, it may not be the only predictor for early recurrence of HCC. This study suggeststhat miR-155 is related to the clinical characaticals of HCC.And it may be a novel diagnostic marker and potential therapeutic target in HCC.

\section{Acknowledgements}

The present study was supported by the Science and Technology Special Reserve Funds, Zhanjiang, China (grant no. Zhang-Ke[2013]125-130).

\section{References}

1. Jemal A, Bray F, Center MM, Ferlay J, Ward E and Forman D: Global cancer statistics. CA Cancer J Clin 61: 69-90, 2011.

2. Ferlay J, Shin HR, Bray F, et al: Estimates of worldwide burden of cancer in 2008: GLOBOCAN 2008. Int J Cancer 127 2893-2917, 2010.

3. Lencioni R, Chen XP, Dagher L and Venook AP: Treatment of intermediate/advanced hepatocellular carcinoma in the clinic: How can outcomes be improved? Oncologist 15 (Suppl 4): S42-S52, 2010.

4. Suh SJ and Yim HJ: Current status of molecular targeted therapies in hepatocellular carcinoma. Korean J Gastroenterol 61: 136-146, 2013 (In Korean).

5. Qin S; Primary Liver Cancer Diagnosis and Treatment Expert Panel of the Chinese Ministry of Health: Guidelines on the diagnosis and treatment of primary liver cancer (2011 edition). Chin Clin Oncol 1:10, 2012.

6. Sanyal AJ, Yoon SK and Lencioni R: The etiology of hepatocellular carcinoma and consequences for treatment. Oncologist 15 (Suppl 4): 14-22, 2010

7. Kumar M, Zhao X and Wang XW: Molecular carcinogenesis of hepatocellular carcinoma and intrahepatic cholangiocarcinoma: One step closer to personalized medicine? Cell Biosci 1: 5, 2011

8. Lee RC, Feinbaum RL and Ambros V: The C. elegans heterochronic gene lin- 4 encodes small RNAs with antisense complementarity to lin-14. Cell 75: 843-854, 1993.
9. Lu J, Getz G, Miska EA, et al : MicroRNA expression profiles classify human cancers. Nature 435: 834-838, 2005.

10. Lagos-Quintana M, Rauhut R, Yalcin A, et al: Identification of tissue-specific microRNAs from mouse. Curr Biol 12: 735-739, 2002.

11. Merhautova, J, Hezova R, Poprach A, et al: miR-155 and miR-484 are associated with time to progression in metastatic renal cell carcinoma treated with sunitinib. Biomed Res Int 2015: 941980, 2015.

12. Ling N, Gu J, Lei Z, et al: microRNA-155 regulates cell proliferation and invasion by targeting FOXO3a in glioma. Oncol Rep 30: 2111-2118, 2013.

13. Zhang GJ, Xiao HX, Tian HP, et al: Upregulation of microRNA-155 promotes the migration and invasion of colorectal cancer cells through the regulation of claudin-1 expression. Int J Mol Med 31: 1375-1380, 2013.

14. Faraoni I, Antonetti FR, Cardone J and Bonmassar E: miR-155 gene: A typical multifunctional microRNA. Biochim Biophys Acta 1792: 497-505, 2009.

15. Macfarlane LA and Murphy PR: MicroRNA: Biogenesis, function and role in cancer. Curr Genomics 11: 537-561, 2010.

16. Papaconstantinou IG, Manta A, Gazouli M, et al: Expression of microRNAs in patients with pancreatic cancer and its prognostic significance. Pancreas 42: 67-71, 2013.

17. Shibuya $H$, Iinuma $H$, Shimada $R$, Horiuchi $A$ and Watanabe $T$ : Clinicopathological and prognostic value of microRNA-21 and microRNA-155 in colorectal cancer. Oncology 79: 313-320, 2010.

18. Huang Z, Huang D, Ni S, Peng Z, Sheng W and Du X: Plasma microRNAs are promising novel biomarkers for early detection of colorectal cancer. Int J Cancer 127: 118-126, 2010.

19. World Medical Association: Declaration of Helsinki. Ethical Principles for Medical Research Involving Human Subjects. http://www.wma.net/en/30publications/10policies/b3/17c.pdf. Accessed December 25, 2011.

20. Tsai HC and Baylin SB: Cancer epigenetics: Linking basic biology to clinical medicine. Cell Res 21: 502-517, 2011.

21. Hu YH, Cai Q and Lan QY: Expression of miR-155 in hepatocellular carcinoma and its effect on the proliferation of hepatocellular carcinoma cells. World J Gastroenterol 19: 1737-1741, 2012.

22. Song CG, Wu XY, Fu FM, et al: Correlation of miR-155 on formalin-fixed paraffin embedded tissues with invasiveness and prognosis of breast cancer. Zhonghua Wai Ke Za Zhi 50: 1011-1014, 2012 (In Chinese).

23. Han ZB, Chen HY, Fan JW, et al: Up-regulation of microRNA-155 promotes cancer cell invasion and predicts poor survival of hepatocellular carcinoma following liver transplantation. J Cancer Res Clin Oncol 138: 153-161, 2012.

24. Michieli P, Chedid M, Lin D, Pierce JH, Mercer WE and Givol D: Induction of WAF1/CIP1 by a p53-independent pathway. Cancer Res 54: 3391-3395, 1994.

25. Xie Q, Chen X, Lu F, et al: Aberrant expression of microRNA 155 may accelerate cell proliferation by targeting sex-determining region $\mathrm{Y}$ box 6 in hepatocellular carcinoma. Cancer 118: 2431-2442, 2012.

26. Yan XL, Jia YL, Chen L, et al: Hepatocellular carcinoma-associated mesenchymal stem cells promote hepatocarcinoma progression: Role of the S100A4-miR155-SOCS1-MMP9 axis. Hepatology 57: 2274-2286, 2013.

27. Shi J, Lai EC, Li N, et al: A new classification for hepatocellular carcinoma with portal vein tumor thrombus. J Hepatobiliary Pancreat Sci 18: 74-80, 2011.

28. Poon RT, Fan ST, Ng IO, et al: Different risk factors and prognosis for early and late intrahepatic recurrence after resection of hepatocellular carcinoma. Cancer 89: 500-507, 2000.

29. Sun J, Jian ZX, Ou YL, Hou BH and Qu JR: Ensured the early recurrent time and model of liver cancer after radical resection. Shi Yong Yi Xue Za Zhi 10: 1748-1750, 2011 (In Chinese).

30. Cai XR, Huang CY, Zhou LY and Zhou HH: Study on risk factors and therapeutic effect of different recurrence period after resection of primary hepatocellular carcinoma. Shi Yong Yi Xue Za Zhi 5: 560-563, 2010 (In Chinese).

31. Braconi C,Henry JC, Kogure T, Schmittgen T and Patel T: The role of microRNAs in human liver cancers. Semin Oncol 38: 752-763, 2011.

32. Shrivastava S, Steele R, Ray R and Ray RB: MicroRNAs: Role in hepatitis C virus pathogenesis. Genes Dis 2: 35-45, 2015.

33. Huang YH, Lin KH, Chen HC, et al: Identification of postoperative prognostic microRNA predictors in hepatocellular carcinoma. PLoS One 7: e37188, 2012. 